\title{
Comparer ce qui est comparable
}

\section{Christoph Bosshard}

Dr méd., vice-président de la FMH, responsable du département Données, démographie et qualité

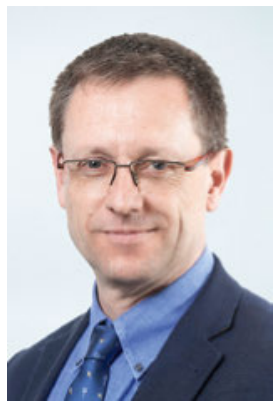

Le bonheur des choses simples: «Ramène un kilo de pain à la maison", entends-je après avoir confirmé que je serai là pour le souper. Devant le rayon, l'affaire se corse: pain blanc ou complet? Avec ou sans graines? Ce qui paraît simple à première vue ne l'est pas toujours. Heureusement, certaines décisions du quotidien n'ont pas d'impact significatif - d'autres en revanche ont bien plus d'importance. Comment évolue la démographie du corps médical? Qu'en est-il du taux d'occupation? Suffit-il d'additionner les têtes pour avoir un bon aperçu de la force de travail des médecins? L'augmentation du travail à temps partiel est un fait de société, y compris chez les médecins, où elle concerne davantage les femmes que les hommes. Si ces derniers sont actuellement plus nombreux à exercer la médecine (59\%, contre $41 \%$ de femmes), cette situation est cependant sur le point de s'inverser durablement: l'avenir de la médecine est clairement féminin, comme le montre le nombre de diplômé-e-s en médecine humaine, qui arriveront sur le marché du travail d'ici six à neuf ans.

\section{La progression du travail à temps partiel va se poursuivre avec la féminisation de la médecine.}

Un autre défi réside dans la définition d'une activité à plein temps, qui n'est pas encore réglée uniformément. A ce sujet, je me souviens encore avoir entendu: «Une journée compte 24 heures, ensuite il y a la nuit, et si cela ne suffit toujours pas, on fera des heures supplémentaires.» Le recensement effectué dans myFMH se base sur des demi-journées travaillées de quatre à six heures. A l'avenir, il s'agira de déterminer si cette méthode tient suffisamment compte de l'hétérogénéité des disciplines, mais aussi des classes d'âges des médecins, afin d'éviter de comparer ce qui n'est pas comparable. Ceci arrive malheureusement bien trop souvent, comme l'illustre un exemple récemment observé dans le canton de Berne: après une vie professionnelle bien remplie, un pédiatre a la chance de trouver une solution pour régler la succession de son cabinet et prendre une retraite bien méritée - cinq médecins à temps partiel se répartissent désormais son travail. La statistique par tête parlerait alors d'une multiplication par cinq des soins.

Au lieu de compter les têtes, les équivalents plein temps sont plus adaptés pour la planification des soins médicaux.

Venons-en aux soins, justement. Outre la perspective des fournisseurs de prestations, il s'agit aussi de considérer la perspective des bénéficiaires. En observant l'évolution démographique de nos concitoyens, nous constatons qu'à l'avenir, la classe d'âge des 75+ va aug menter de manière disproportionnée par rapport au reste de la population. Cette évolution est également au centre du débat actuel sur la prévoyance vieillesse. La remise en question du système de solidarité déjà parmi les personnes en bonne santé est indéniable. Cette situation est encore aggravée par la part des coûts de santé de la classe $75+$, nettement supérieure à celle des autres classes. Nous sommes là en présence de deux facteurs disproportionnés à effet multiplicateur du côté des bénéficiaires de soins, qui auront dans tous les cas un impact sur les soins à l'avenir, même si les besoins individuels de la population n'évoluent pas. Il appartient aux milieux politiques et administratifs de présenter des perspectives. Du côté des médecins, la FMH prend ses responsabilités et met tout en œuvre pour que notre système de santé tienne le cap. J'en profite pour adresser mes plus vifs remerciements à tous nos membres qui apportent leur pierre à l'édifice en mettant à jour leurs données sur myFMH. 